\title{
A catalog of the types of craneflies (Limoniidae and Tipulidae) (Insecta, Diptera) deposited in the Museo Argentino de Ciencias Naturales, Buenos Aires
}

\begin{abstract}
Axel O. BACHMANN
División Entomología, Museo Argentino de Ciencias Naturales, Av. A. Gallardo 470, C1405DJR Buenos Aires Argentina. Departamento de Biodiversidad y Biología Experimental, Facultad de Ciencias Exactas y Naturales, Universidad de Buenos Aires, Ciudad Universitaria, C1428EHA. Argentina. bachmann@bg.fcen.uba.ar.

Abstract: The type specimens of all accepted categories of twenty-eight names of craneflies (twenty-two of Limoniidae and six of Tipulidae), housed in this Museum are recorded. Specimens labeled as types, but which are not types, or are only very dubiously acceptable as types, are recorded in a separate list, in order to assist in future research. Most of the species here catalogued were described by Dr. C.P. Alexander, based upon specimens from this Museum, sent to him by C. Bruch.
\end{abstract}

Key words: Insecta. Diptera, Craneflies, Limoniidae, Tipulidae, type specimens.

Resumen: Catálogo de los tipos de típulas (Limoniidae y Tipulidae) (Insecta, Diptera) depositados en el Museo Argentino de Ciencias Naturales, Buenos Aires. Se catalogan los ejemplares típicos de todas las categorías aceptadas, de veintiocho nombres de típulas (veintidós de Limoniidae y seis de Tipulidae). Ejemplares rotulados como tipos, pero que no son tipos, o que solo muy dudosamente serían aceptables como tipos, se incluyen en una lista separada, para facilitar futuras investigaciones. La mayoría de las especies aquí catalogadas fue descrita por el Dr. C.P. Alexander, sobre la base de ejemplares del Museo, enviados por C. Bruch.

Palabras clave: Insecta, Diptera, Típulas, Limoniidae, Tipulidae, ejemplares tipo.

\section{PRESENTATION}

A catalog of the type specimens of craneflies, Limoniidae and Tipulidae, housed in the Entomological Division of the Museum is presented, referred to twenty-eight names. Most of the species were described by Dr. C.P. Alexander, based upon specimens from this Museum sent to him by C. Bruch; the identification labels were handwritten by Bruch, copied from letters, and not by Alexander himself. Bruch used to put red framed labels for the specimens, and pale green labels reading "Typus" or "Cotypus"; the cotypus labels seem to have been attached sometimes to syntypes and sometimes to paratypes, making the interpretation uncertain. In several cases it is not possible to conclude about the type category, as the specimens were labelled "Typus", and the whole series bear identical locality labels. In such situations, I leave the question open to future research by specialists, writing for example "Holot.? parat.?" (perhaps some details in the descriptions will sometimes help?). Although several specimens bear a small piece of paper, cut from a handwrit- ten letter, probably written by Alexander, this fact does not assure that Alexander examined and labeled just that specimen.

When no name bearing specimen(s) was (were) formally designated, and the amount of specimens examinated was not mentioned, it is assumed that there was a series of syntypes (recommendation $73 \mathrm{~F}$ of the International Code of Zoological Nomenclature, 1999), eventually "single syntypes".

In geographical mentions, if the country is not stated, Argentina is meant.

Specific and subspecific epithets are alphabetically entered in a single list, with a brief bibliographic quotation, localities, and a mention of types, if these were stated. An account of the type specimens actually here housed follows, with a description of all the labels. Taxionomical and nomenclatorial updating is added, following Alexander \& Alexander (1970), and Oosterbroek (2010).

Some specimens are labeled as types, but were not mentioned in the original descriptions, and/or were collected and designated later, and published or not; they are not acceptable as types, but in or- 
der to assist in future research, they are recorded in a separate list, below.

All specimens bear a small inverted label with the entry in the database of the Entomological Division of the Museum, as "MACN-En 0000". Specimen information is currently managed with the application "Aurora" (Rodríguez, 2007-2010).

I am sincerely thankful to Miss Priscila Hanschel for kindly help with the data base management.

Abbreviations used: allot., allotype; coll., collection; det., determinated (identified); handwr., handwritten; holot., holotype; parat., paratype(s); print., printed; Prov., province(s); spec., specimen(s); synt., syntype(s).

\section{CATALOG OF THE TYPES}

\section{LIMONIIDAE}

aequabilis [Geranomyia (Geranomyia)]. Alexander 1923b. 311. Córdoba Prov.: Alta Gracia: La Granja

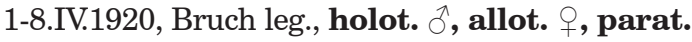
one $\hat{\sigma}$, one $q$.

Holot.? parat.? § pinned, "La Granja (Alta Gracia) / Prov. de Córdoba / 1-8.IV-1920. C. Bruch" photographed; " 13 " handwr.; "Typus" print. on pale green paper; "Geranomyia / aequabile / Alex." handwr. by Bruch, red frame. "MACN-En 7948" print., reverse.

Notes: The "Typus" label suggests it is the holot. Recorded as Limonia (Geranomyia) aequabilis aequabilis by Alexander \& Alexander (1970: 60), and as Geranomyia aequabilis by Oosterbroek (2010).

annulicornis [Epiphragma]. Alexander 1921a: 176-177. Tucumán Prov.: Quebrada Famaillá 1600 m, 16.X.1920, leg. Weiser, holot. $\hat{0}$, allot. + , three parat. $\hat{\jmath}$,, . Tucumán Prov.: Sauce Gaucho (sic), $500 \mathrm{~m}$ 12.X.1920, leg. Weiser, parat. one 9 .

Holot.?, parat.? pinned, "Quebrada Fa- / maillá 15.X.920. / Tucumán / Weiser" handwr. by Bruch. One parat., "Sauce Quacho (sic) / Tucumán / Weiser" handwr. by Bruch. Both: "61" handwr.; "Typus" print. on pale green paper; "Epiphragma / annulicornis / Alex." handwr. by Bruch, red frame. "MACN-En 7936 [7937]" print., reverse.

Notes: The "Typus" label suggests it is the holot. The date differs slightly, but otherwise the labels seem authentic. Recorded under Epiphragma by Alexander \& Alexander (1970: 95), and under Epiphragma (Epiphragma) by Oosterbroek (2010).

argentinensis [Cryptolabis (Procryptolabis)]. Alexander 1923a: 184. Córdoba Prov.: Alta Gracia:
La Granja 1-8.IV.1920, leg. Bruch, holot. ${ }^{\circ}$, allot. 우 (mounted together), four parat. $\hat{~}$, $q$.

Holot.?, parat.? in double mounting with a minutie on a card, "La Granja (Alta Gracia) / Prov. de Cordoba / 1-8.IV.1920. C. Bruch" photographed; "17" handwr.; "Typus" print. on pale green paper; "Cryptolabis / (Procryptolabis) / argentinensis / Alex." handwr. by Bruch, red frame. "MACN-En 7949" print., reverse.

Notes: The "Typus" label would suggest it is the holot., but the holot. and the allot. are said to be mounted together, which is not the case. Recorded as Cryptolabis (Procryptolabis) argentinensis by Alexander \& Alexander (1970: 122), and by Oosterbroek (2010).

argentinensis [Geranomyia]. Alexander 1920b: 1-2. Buenos Aires Prov., leg. Bruch, holot. $\widehat{0}$, allot. $\circ$, five "paratopotypes" $\hat{\circ}, \circ$.

One parat. $q$ pinned, "Rep. Argentina / Prov. Buenos Aires / 190_ / C. Bruch" print., black frame; "Typus" print. on pale green paper; "Geranomyia / argentinensis / Alex." handwr. by Bruch, red frame; "Paratype [print.] / Geranomyi / argentinens [handwr. by Alexander?]" on green paper, cut "MACN-En 7950" print., reverse.

Notes: The cut label was probably written on a letter by Alexander. Recorded under Limonia (Geranomyia) by Alexander \& Alexander (1970: 60 ), and under Geranomyia by Oosterbroek (2010).

basalis [Rhabdomastix (Sacandaga)]. Alexander 1921b: 293-294. Catamarca Prov.: Punta de Balasto, 2300 m, 10-11.XI.1920, leg.Weiser, holot. $\delta^{7}$, allot. + . Catamarca Prov.: Santa María 30.X.1920, leg. Weiser, parat. one $\delta$, three + . Mendoza Prov.: Diamante Stream I.1921 leg. Carette, parat. one $\hat{\jmath}$, one $q$. Tucumán Prov.: Bañado, Santa María Valley 2-11.IV.1921, leg. Weiser, parat. sixty $\hat{\rho}$, $q$.

Three parat. in double mounting with minutien on cards, "Bañado / Tucum. Weiser [Tucum. 6.IV.921, Tucum. V.921]" handwr. by Bruch; "Typus" ["Cotypus", "Cotypus"] handwr. by Bruch on pale green paper; on two of them "Rhabdomastix / basalis

Alex." handwr. by Bruch, remainings of a black frame. To the 3d one,"cotypus" I add "Rhabdomastix / basalis Alex." handwr. "MACNEn 7951, [7952, 7953]" print., reverse.

Note: Recorded as Cheilotrichia (Empeda) basalis by Alexander \& Alexander (1970: 119), and by Oosterbroek (2010).

bruchi [Gonomyia (Leiponeura)]. Alexander 1920a: 217-219. Buenos Aires Prov. 11.X.1919, 
leg. Bruch, holot. $\delta^{1}$. Córdoba Prov.: Alta Gracia, La Aranja (sic, pro La Granja) 1-8.IV.1920, leg. Bruch, one $\widehat{\partial}$, two $\odot$ (probably intended as parat., but not formally designated as such).

One parat.? in double mounting, with a minutie on a card, "La Granja (Alta Gracia) / Prov. de Córdoba / 1-8.IV-1920. C. Bruch" photographed; "9" handwr.; "Typus" print. on pale green paper; "Gonomyia / (Leiponeura) / Bruchi / Alex." handwr. by Bruch, red frame. "MACN-En 7954" print., reverse.

Notes: Probably intended as a paratype, but not formally designated as such. Recorded under Gonomyia (Lipophleps) by Alexander \& Alexander (1970: 135), and under Gonomyia (Leiponeura) by Oosterbroek (2010).

bruchi [Molophilus]. Alexander 1923a: 182. Buenos Aires Prov.: La Plata: Palo Blanco 24.IV.1920 leg. Bruch, holot. $\hat{\text { s, many parat. }}$.

Holot.?, one parat.? in double mounting, with a minutie on a card, "Palo Blanco / La Plata / 2.V.920 C.B." handwr. by Bruch; "27" handwr.; "Typus" print. on pale green paper; "Molophilus / Bruchi / Alex." handwr. by Bruch, red frame. "MACN-En 7955" print., reverse.

Notes: The collecting dates differ slightly, but otherwise the labels seem authentic. The "Typus" label suggests it is the holot. Recorded under Molophilus (Molophilus) by Alexander \& Alexander (1970: 145), and by Oosterbroek (2010).

cladophoroides [Erioptera (Erioptera)]. Alexander 1921c: 162-163. Buenos Aires Prov.: La Plata 13.X.1920 leg. Bruch, holot. ô, allot. ; 13-18.X.1920, leg. Bruch, seven parat. $\hat{\jmath}$, + .

Holot. + three parat.?, four parat.? pinned, "Rep. Argentina / Prov. Buenos Aires / 4.X.1920 [10.X.1920, 10.X.1920, 190_] / C. Bruch" print., dates handwr., black frame; " 58 " handwr.; "Typus" print. on pale green paper; on one, also "Foto" print. on pale green paper; "Erioptera (E.) / cladophoroides / Alex." handwr. by Bruch, red frame. "MACNEn 7956 [7957, 7958, 7959]" print., reverse.

Notes: The collecting dates differ slightly, but otherwise the labels seem authentic. The "Typus" label render the type category uncertain. Recorded under Erioptera (Erioptera) by Alexander \& Alexander (1970: 123), and by Oosterbroek (2010).

flavofascialis [Dicranomyia]. Alexander 1924: 61-62. Córdoba Prov.: Alta Gracia: La Granja 1-8. IV.1920, leg. Bruch, holot. $\delta$, allot. + , thirty parat. $0,+$.

Holot. + allot.?, two parat. $\partial^{1}, \uparrow^{?}$ pinned, "La Granja (Alta Gracia) / Prov. de Córdoba / 1-8. IV.1920. C. Bruch" photographed; "Typus" print. on pale green paper; "Dicranomyia / flavofascialis / Alex." handwr. by Bruch, red frame, the of also "7" handwr. "MACN-En 7960 [7961]" print., reverse.

Notes: The "Typus" labels render it uncertain if they are holot. + parat., or two parat. Recorded under Limonia (Dicranomyia) by Alexander \& Alexander (1970: 53), and under Dicranomyia (Dicranomyia) by Oosterbroek (2010).

fuscivena [Erioptera (Mesocyphona) immaculata]. Alexander 1923b: 311. Córdoba Prov:: Alta Gracia: La Granja 1-8.IV.1920 leg. Bruch, holot. ${ }^{\wedge}$, allot. + , parat. five $\delta$.

Holot. + one parat. $\delta$ ?, two parat. $\delta$ ? in double mounting with minutien on cards, "La Granja (Alta Gracia) / Prov. de Córdoba / 1-8.IV-1920. C. Bruch" photographed; "Typus" print. on pale green paper; "Erioptera (M.) [E. (Mesocyphona)] / immaculata Al. [Alex.] / v. fuscivena / Alex." handwr. by Bruch, red frame, one of them also: " 16 " handwr. "MACN-En 7962 [7963]" print., reverse.

Notes: The "Typus" labels render it uncertain if they are holot. + parat., or two parat. Recorded as Erioptera (Mesocyphona) immaculata ssp. fuscivena by Alexander \& Alexander (1970: 124), and by Oosterbroek (2010).

globulicornis [Dicranomyia]. Alexander 1924: 63-64. Jujuy Prov.: Tilcara 20.III.1920 leg. Weiser, holot. $\hat{\jmath}$, one parat. $\hat{\delta}$.

One parat. $\circlearrowleft$ pinned, “Tilcara / Jujuy / Weiser / 20.III.920" handwr. by Bruch; "Paratype" print. on green paper; "Dicranomyia / globulicornis / Alex." handwr. by Bruch, red frame. "MACN-En 7964" print., reverse.

Note: Recorded under Limonia (Dicranomyia) by Alexander \& Alexander (1970: 53) and under Dicranomyia (Dicranomyia) by Oosterbroek (2010).

honestus [Molophilus]. Alexander 1923a: 181-182. Córdoba Prov.: Alta Gracia: La Granja 1-8.IV.1920, leg. Bruch, holot. $\delta$, allot. + , parat. two ${ }^{\dagger}$.

Holot. ${ }^{\top}$ ?, one parat. ${ }^{\lambda}$ ? pinned, "La Granja (Alta Gracia) / Prov. de Córdoba / 1-8.IV.1920. C. Bruch" photographed; "14" handwr.; "Typus" print. on pale green paper; "Molophilus / honestus / Alex." handwr. by Bruch, red frame. "MACN-En 7965" print., reverse.

Notes: The "Typus" label suggests it is the holot. Recorded under Molophilus (Molophilus) by Alexander \& Alexander (1970: 147), and by Oosterbroek (2010).

misera [Gonomyia (Leiponeura)]. Alexander 1921c: 164-165. Tucumán Prov.: Famaillá Stream $400 \mathrm{~m}$ 12.X.1920, leg. Weiser, holot. ${ }^{\top}$, in author's coll., allot. + , eight parat. $\hat{\delta}$, $\circ$. 
Allot. $q$ + one parat.?, two parat.? pinned, "Arr ${ }^{\circ}$ Famaillá / Tucumán / 500 m. 12.X.20 / Weiser" handwr. by Bruch; "Typus" print. on pale green paper; "66" handwr., one of them, also "Gonomyia / (Leiponeura) / misera Alex." handwr. by Bruch, red frame. Two parat.?, pinned "Quebrada Fa- / maillá 16.X.20 / Tucumán / Weiser" handwr. by Bruch; "66" handwr., "Cotypus" print. on pale green paper; on one, also "Gonomyia / (Leiponeura) misera / Alex." handwr. by Bruch. I add copies of the last label to the other two. "MACN-En 7972 [7973, 7974, 7975]" print., reverse.

Notes: Recorded under Gonomyia (Lipophleps) by Alexander \& Alexander (1970: 137), and under G. (Liponeura) by Oosterbroek (2010). The spec. labeled "Cotypus" suggest they are parat.

ogloblini [Hexatoma (Eriocera)]. Alexander 1935: 325-326. Misiones Prov.: Loreto, leg. Ogloblin, holot. $\odot$. One parat. $\odot$ in Bruch coll.

One parat. + on a large card, «Est. Exp. Loreto / (Misiones, Arg.) / Dr. A.A. Oglobin (sic, pro Ogloblin)" print.; "Paratypus" handwr. on pale green paper; "Hexatoma / (Eriocera) / Ogloblini / Alex." handwr. by Bruch. "MACN-En 7976" print., reverse.

Note: Recorded as Hexatoma (Eriocera) ogloblini by Alexander \& Alexander (1970: 103), and by Oosterbroek (2010).

omissivena [Dicranomyia]. Alexander 1922: 207. Tucumán Prov.: Quebrada Famaillá 16.X.1920, leg. Weiser, holot. $\delta^{\lambda}$, allot. $\odot$, three parat. $\delta$,, .

Two spec. $\partial$,, , holot.?, allot.? parat.? pinned, "Quebrada Fa- / maillá 16.X.20 / Tucumán / Weiser" handwr. by Bruch; "62" handwr.; "Typus" print. on pale green paper; at the $\delta$ : "Dicranomyia / omissivena / Alex." handwr. by Bruch, red frame. I add a copy of the last label to the o. "MACN-En 7977 [7978]" print., reverse.

Notes: The "Typus" label suggests that the $\delta$ is the holot. Recorded under Limonia (Dicranomyia) by Alexander \& Alexander (1970: 55), and under Dicranomyia (Dicranomyia) by Oosterbroek (2010).

patruelis [Dicranomyia]. Alexander 1924: 62-63. Córdoba Prov.: Alta Gracia: La Granja 1-8. IV.1920, leg. Bruch, holot. ô, allot.

Holot. ${ }^{\lambda}$, pinned, "La Granja (Alta Gracia) / Prov. de Córdoba / 1-8.IV.1920. C. Bruch" photographed; "Typus" print. on pale green paper; "Dicranomyia / patruelis / Alex." handwr. by Bruch, red frame. "MACN-En 7979" print., reverse.
Note: Recorded under Limonia (Dicranomyia) by Alexander \& Alexander (1970: 56), and under Dicranomyia (Dicranomyia) by Oosterbroek (2010).

platensis [Geranomyia (Geranomyia)]. Alexander 1923b: 310-311. Buenos Aires Prov:: La Plata IV.1920, leg. Durione, holot. $\hat{\jmath}$, allot. + , parat. two + .

Allot. $\odot$ ?, parat. $\odot$ ?, pinned, "La Plata IV.920 / Durione" handwr. by Bruch; "35" handwr.; "Typus" print. on pale green paper; "Geranomyia / platensis / Alex." handwr. by Bruch, red frame. There are two further spec., that might be parat.: "La Plata / muro Lago / 14.V.920 C.B." handwr. by Bruch; "35" handwr.; "Typus" ["Cotypus"] print. on pale green paper, one of them "Geranomyia / platensis..." as the former; to the other I add a copy of this label. "MACN-En 7980 [7981, 7982]" print., reverse.

Notes: The "Typus" and "Cotypus" labels suggest they are the allot. and one parat. Recorded under Limonia (Geranomyia) by Alexander \& Alexander (1970: 65) and under Geranomyia by Oosterbroek (2010).

platensis [Limnophila]. Alexander 1923a: 184185. Buenos Aires Prov.: La Plata: Palo Blanco, 18.IV.1920, leg. Bruch, holot. O’ $^{\text {; } 2 . V .1920 ~ a l l o t . ~}$ ; 18-24.IV.1920 parat. five $\delta$.

Holot. 0 ?, parat. $\delta$ ? pinned, "Palo Blanco / La Plata / 18.IV.920 C.B." handwr. by Bruch; "25" handwr.; "Typus" print. on pale green paper; "Limnophila / platensis / Alex." handwr. by Bruch, red frame. "MACN-En 7983" print., reverse.

Notes: The "Typus" label suggests it is the holot. Recorded under Paralimnophila by Alexander \& Alexander (1970: 110) and under Austrolimnophila (Austrolimnophila) by Oosterbroek (2010).

sanctaecruzae (sub sanctae-cruzae) [Dicranomyia]. Alexander 1920b: 3-4. Santa Cruz Prov.: Tunel Valley, leg. Witte, holot. $\partial^{\top}$, allot. + , parat. one $q$. Tierra del Fuego Prov., leg. Ohlin, one parat. $P$ in Mus. Stockholm (Sweden).

One parat. pinned, "Valle Túnel / Santa Cruz" handwr. by Bruch; "Typus" print. on pale green paper; "23" handwr.; "Dicranomyia / Sanctae-Cruzae / Alex." handwr. by Bruch, red frame; "Paratype" [print.] / Dicranomyi / Sanctae cru [handwr.]" on green paper, cut from a letter?, inverted, with "C.P. Ale" at the lower margin. "MACN-En 7939" print., reverse.

Notes: The cut label was probably written by Alexander. Recorded under Limonia (Dicranomyia) by Alexander \& Alexander (1970: 57), and under Dicranomyia (Dicranomyia) by Oosterbroek (2010). 
saxicola [Gonomyia (Progonomyia)]. Alexander 1923a: 182-183. Córdoba Prov.: Alta Gracia: La Granja 1-8.IV.1920, leg. Bruch, holot. ô, allot. $\circ$, many parat. 0 , $\circ$.

Two spec., holot.?, allot.? parat.?, one pinned, one on double mounting with a minutie on a card, "La Granja (Alta Gracia) / Prov. de Córdoba / 1-8.IV.1920. C. Bruch" photographed; "Typus" print. on pale green paper, one of them also "8" handwr. "MACN-En 7990 [7991]" print., reverse.

Notes: The "Typus" label renders the type category uncertain. Recorded under Gonomyia (Progonomyia) by Alexander \& Alexander (1970:142), and under Ellipteroides (Progonomyia) by Oosterbroek (2010).

serotina [Geranomyia (Geranomyia)]. Alexander 1923b: 309-310. Córdoba Prov.: Alta Gracia: La Granja 1-8.IV.1920, leg. Bruch, holot. ô, allot. $\circ$, five parat. $\hat{\delta}$, + .

Allot. $\odot$ ?, one parat. $\odot$ ? pinned, "La Granja (Alta Gracia) / Prov. de Córdoba / 1-8.IV.1920. C. Bruch" photographed; "12" handwr.; "Typus" print. on pale green paper; "Geranomyia / serotina / Alex." handwr. by Bruch, red frame. "MACN-En 7992" print., reverse.

Note: The "Typus" label suggests it is the allot. Recorded under Limonia (Geranomyia) by Alexander \& Alexander (1970: 66), and under Geranomyia by Oosterbroek (2010).

weiseri [Gonomyia (Gonomyella)]. Alexander 1920a: 215-216. Jujuy Prov.: Cueva Iturbe 3700

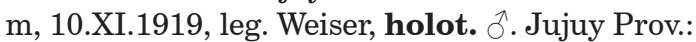
Hornadita 3400 m 30.XI.1919, leg. Weiser, allot. , parat. one ${ }_{\text {. }}$. Jujuy Prov.: Tilcara 20.III.1920, parat. seven $\delta$, four $q$.

Allot. $q$ ?, one parat. $q$ ? pinned, "Rep. Argentina / Prov. Jujuy / 30.XI.1919 / C. Bruch" print., date handwr., black frame; "Hornadita / 3400 m. / Weiser" handwr. by Bruch; "Typus" print. on pale green paper; " 33 " handwr.; "Gonomyia / Weiseri / Alex." handwr. by Bruch, red frame. "MACN-En 7995" print., reverse.

Note: The "Typus" label suggests it is the allot. Recorded under Gonomyia (Progonomyia) by Alexander \& Alexander (1970: 142), and under Ellipteroides (Progonomyia) by Oosterbroek (2010).

\section{TIPULIDAE}

andina [Tipula]. Brèthes 1909: 88-89. Mendoza Prov.: Puente del Inca I.1908, one + .

Holot. + pinned, with only left hind leg, left wing damaged, "10361" handwr., red frame; "Mendoza" handwr. on green paper, black frame, reverse white; "Tipula / andina Brèthes" handwr. by Brèthes. "MACN-En 7935" print., reverse.

Notes: The specimen lacks a type label, making the type condition doubtful. In the register book of the Entomology Division, the entry 10361 reads Mendoza, Cordillera, dádiva Wolffhugel [a gift from Wolffhügel]. Recorded as Tipula (Eumicrotipula) andina by Alexander \& Alexander (1970: 21) and by Oosterbroek (2010).

australis [Brachypremna]. Alexander 1923a: 185-186. Córdoba Prov.: Alta Gracia: La Granja, leg. Bruch, holot. $\overbrace{}^{\lambda}$, allot. $\circ$, several parat. $\partial^{\lambda},+$.

Two spec., holot. + parat.?, two parat.? pinned, one with a leg glued to a card, "La Granja (Alta Gracia) / Prov. de Córdoba / 1-8.IV.1920. C. Bruch” print.; “Typus” print. on pale green paper; "Brachypremna / australis / Alex." handwr. by Bruch, red frame. "MACN-En 7938 [7939]" print., reverse.

Note: The "Typus" label renders the type category uncertain. Recorded as Brachypremna australis by Alexander \& Alexander (1970: 6), and by Oosterbroek (2010).

barretoi [Tipula]. Alexander 1923b: 312-313. Buenos Aires Prov.: Monte Veloz III.1920, leg. Barreto, holot. $\delta^{\lambda}$, allot. $\odot$, parat. four $\lambda$.

Holot. $\delta$ + allot. $q$ ?, allot. $q+$ parat. $\delta$ ? pinned, "Monte Veloz / Bs. Aires / B. Barreto" handwr. by Bruch; "21" handwr.; "Typus" ["Cotypus"] print. on pale green paper; "Tipula / Barretoi / Alex." handwr. by Bruch, red frame [remainings of a black frame]. "MACN-En 7940 (ठ) $[7941($ ( ) $)] "$ print., reverse.

Notes: The "Typus" and "Cotypus" labels suggest they may be the holot. and allot. Recorded as Tipula (Eumicrotipula) barretoi by Alexander \& Alexander (1970: 22), and by Oosterbroek (2010).

bruchi [Tipula]. Alexander 1920b: 10-11. Buenos Aires Prov., leg. Bruch, holot. $\widehat{\jmath}$, parat. two $\widehat{\partial}$.

One parat. pinned, "Rep. Argentina / Prov. Buenos Aires / 190_ / C. Bruch" print., black frame; "Cotypus" print. on pale green paper; "Tipula / Bruchi / Alex." handwr. by Bruch, red frame. "MACN-En 7942" print., reverse.

Notes: The "Cotypus" label suggests it is a parat. Recorded as Tipula (Eumicrotipula) bruchi by Alexander \& Alexander (1970: 23) and by Oosterbroek (2010).

jujuyensis [Holorusia]. Alexander 1920a: 220221. Jujuy Prov.: Jujuy City 12.I.1920 leg. Weiser, holot. $\hat{\jmath}$, parat. one $\hat{\jmath}$, one $\phi$. Tilcara 12.I.1920, Weiser leg., allot. . Córdoba Prov.: Alta Gracia 1-8.IV.1920 Bruch leg., parat. ten ${ }^{1}$, two + . 
Two parat. pinned, "La Granja (Alta Gracia) / Prov. de Córdoba / 1-8.IV.1920 C. Bruch" print.; "Cotypus" print. on pale green paper; "Holorusia / jujuyensis / Alex." handwr. by Bruch, red frame; one of them, also " 3 " handwr. "MACN-En 7943 [7944]" print., reverse.

Note: Recorded as Ischnotoma (Icriomastax) jujuyensis by Alexander \& Alexander (1970: 9), and by Oosterbroek (2010).

moniliferoides [Tipula]. Alexander 1920a: 221. Buenos Aires Prov.: Monte Veloz, 17.I.1920, leg.

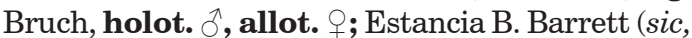
pro Barreto) 14-17.I.1920 leg. Bruch, parat. two ๙ , five + . Córdoba Prov.: Alta Gracia: La Granja, leg. Bruch, parat. four $\delta^{\lambda}$, four $q$.

Three spec., one $\widehat{\jmath}$, two $\odot$, holot.?, allot.?, parat.?, pinned, "Rep. Argentina / Prov. Buenos Aires / 14.I.1920 [10.II.1920] / C. Bruch" print., dates handwr., black frame, two of them $(\hat{\partial},+$ ) : "Estancia / B. Barreto / Monte Veloz" handwr. by Bruch, red frame; "Typus" [on two] "Cotypus" [on one] print. on pale green paper; "Tipula / moniliferoides / Alex." handwr. by Bruch, red frame. "MACN-En 7945 [7946, 7947]" print., reverse.

Notes: The third spec. lacks a locality label; it should perhaps not even be a type. Recorded under Tipula (Eumicrotipula) by Alexander \& Alexander (1970: 27), and by Oosterbroek (2010).

\section{SPECIMENS LABELLED AS TYPES BUT NOT MENTIONED IN THE ORIGINAL PUBLICATION}

\section{LIMONIIDAE}

jorgenseni [Dicranomyia]. Alexander 1919: 134, pl. XVII: f. 3. Catamarca Prov.: Esquina Grande 1.V.1915, leg. Jörgensen, holot. ${ }^{\lambda}$, allot. $\bigcirc$; Cerro de Carocal 3100 m 17.II.1915, leg. Jörgensen, parat. one $\hat{t}$.

Two spec. pinned "Pueblo Viejo / Rio San Juan / Jujuy 3680 m / Weiser 17.IV.920" handwr. by Bruch; " 55 " handwr.; "Cotypus" print. on pale green paper; at one: "Dicranomyia / Jörgenseni / Alex." handwr. by Bruch, red frame. I add a copy of the last label to the other spec. "MACN-En 7966 [7967]" print., reverse.

Notes: Jujuy was not mentioned in the original publication; moreover the spec. were collected later. Recorded as Limonia (Dicranomyia) jorgenseni by Alexander \& Alexander (1970: 54), and under Dicranomyia (Dicranomyia) by Oosterbroek (2010).

jujuyensis [Dicranomyia]. Alexander 1924: 64-65. Jujuy: Tilcara 20.III.1920, leg. Weiser, holot. $\partial^{\lambda}$.
Four spec. pinned, "Rep. Argentina / Prov. Jujuy / 22.V.1920 / C. Bruch" print., date handwr., black frame; "Ciudad / Weiser" handwr. by Bruch; "48" handwr.; "Typus" [on three]; "Cotypus" [on one] print. on pale green paper; "Dicranomyia / jujuyensis / Alex." [on two] handwr. by Bruch, red frame. I add a copy of the last label to the other two. "MACN-En 7968 [7969, 7970, 7971]" print., reverse.

Notes: Only the holot. from Tilcara was mentioned. The "Typus" and "Cotypus" labels are puzzling. Recorded under Limonia (Dicranomyia) by Alexander \& Alexander (1970: 54), and under Dicronomyia (Dicranomyia) by Oosterbroek (2010).

regifica [Dicranomyia]. Alexander 1916: 5-6. Perú: Matucana 7788 feet, leg. Parish, holot. ô, allot. ${ }^{\circ}$. Perú: Jauja 11878 feet, leg. Parish, parat. three $\hat{o}$.

Five spec. pinned, "Pueblo Viejo / Rio San Juan [on two] Santa Catalina [on three] / Jujuy $3680 \mathrm{~m}$ [on two] / Weiser 17.IV.920 [on two] 2.IV.920 [on three]" handwr. by Bruch; "Typus" print. on pale green paper; " 54 " handwr.; "Dicranomyia / regifica / Alex" [on three] handwr. by Bruch, red frame. I add copies of the last label to the other two. "MACN-En 7934 [7935, 7936, 7937, 7938]" print., reverse.

Notes: Two localities in Perú, but none in Argentina were mentioned. Recorded under Limonia (Dicranomyia) by Alexander \& Alexander (1970: 57), and under Dicronomyia (Dicranomyia) by Oosterbroek (2010).

subfuscata [Limnophila]. Alexander 1921a: 177-178. Buenos Aires Prov. 10.X.1920, leg. Bruch. Holot. $\widehat{o}$ in Mus. La Plata.

One spec., pinned, "Palo Blanco / La Plata / 20.XI.920" handwr. by Bruch; "59" handwr.; "Typus" print. on pale green paper; "Limnophila / subfuscata / Alex." handwr. by Bruch, red frame. "MACN-En 7993" print., reverse.

Notes: Only the ${ }^{\widehat{\lambda}}$ holot., in the Museo de La Plata, was mentioned; the dates differ slightly. Recorded under Paralimnophila by Alexander \& Alexander (1970: 110), and under Paralimnophila (Paralimnophila) by Oosterbroek (2010).

subterminalis [Rhipidia]. Alexander 1921a: 175. Buenos Aires Prov.: La Plata, Bosque, leg. Bruch, 23.V.1920, holot. $\bigcirc$ in Museo de La Plata.

One spec. pinned, "Rep. Argentina / Prov. Buenos Aires / 21.X.1920 / C. Bruch" print., date handwr., black frame; " 45 " handwr.; "Typus" and "Foto" print. on pale green paper; "Rhipidia / subterminalis / Alex." handwr. by Bruch, red frame. "MACN-En 7994" print., reverse. 
Notes: Only the holot. @ in the Museo de La Plata was mentioned. Recorded under Limonia (Rhipidia) by Alexander \& Alexander (1970: 78), and under Rhipidia (Rhipidia) by Oosterbroek (2010).

\section{BIBLIOGRAPHY}

Alexander, C.P. 1916. New or little-known crane-flies from Colombia, Ecuador and Peru (Tipulidae, Diptera). Transactions of the American Entomological Society 42: 1-32, pl. I-V.

Alexander, C.P. 1919. Records and descriptions of Neotropical crane-flies (Tipulidae, Diptera). I. Journal of the New York Entomological Society 27 (3-4): 132-154, pl. XVII: f. 1-16.

Alexander, C.P. 1920a. Undescribed crane-flies from Argentina (Tipulidae, Diptera). Entomological News 31 (8): 215-221.

Alexander, C.P. 1920b. Records and descriptions of Neotropical crane-flies (Tipulidae, Diptera). II. Journal of the New York Entomological Society 28 (1): 1-13.

Alexander, C.P. 1921a. Undescribed crane-flies from Argentina (Tipulidae, Dipt.). Part III. Entomological News 32 (6): 175-179.

Alexander, C.P. 1921b. Undescribed crane-flies from Argentina (Tipulidae, Diptera). Part IV. Entomological News 32 (10): 292-295.

Alexander, C.P. 1921c. Undescribed Eriopterine crane-flies from Argentina (Tipulidae, Diptera). Canadian Entomologist 53 (7): 162-166.

Alexander, C.P. 1922. Undescribed crane-flies from Argentina (Tipulidae, Dipt.). Part V. Entomological News 33 (7): 207-210.
Alexander, C.P. 1923a. Undescribed crane-flies from Argentina (Dipt.: Tipulidae). Part VI. Entomological News 34 (6): 181-186.

Alexander, C.P. 1923b. Undescribed crane-flies from Argentina (Dipt.: Tipulidae). Part VII. Entomological News 34 (10): 309-313.

Alexander, C.P. 1924. Undescribed crane-flies from Argentina (Dipt.: Tipulidae). Part VIII. Entomological News 35 (2): 61-65.

Alexander, C.P. 1935. New species of crane-flies from South America. Part VI. Annals of the Entomological Society of America 28 (3): 313-331.

Alexander, C.P. \& Alexander, M.M. 1970. Family Tipulidae. A Catalogue of the Diptera of the Americas South of the United States (4): 4.1-4.259. Univ. São Paulo, Mus. Zool.

Brèthes, J. 1909. Dípteros é himenópteros de Mendoza. Anales del Museo Nacional, Buenos Aires 19 [ $3^{\mathbf{a}}$ ser. 12]: 85-105, f. 1-3.

International Commission on Zoological Nomenclature (ICZN). 1999. International Code of Zoological Nomenclature, 4th. ed.: XIX + 306 p.

Oosterbroek, P. 2010. Catalogue of the craneflies of the World (Diptera: Tipuloidea: Pediciidae, Limoniidae, Cylindrotomidae, Tipulidae).http://ip30.eti.uva.nl/ $c c w$. Last updated 17.VI.2010.

Rodríguez, D. 2007-2010. Aurora. Aplicación para el manejo de datos de colecciones biológicas. Museo Argentino de Ciencias Naturales "Bernardino Rivadavia", Consejo Nacional de Investigaciones Científicas y Técnicas, CONICET.

Recibido: 20/XII/2010 Aceptado: 22/VI/2011 
